\title{
Recognition and Prevention of Fetal Alcohol Syndrome
}

\author{
R. Louise Floyd, DSN, RN, Mary J. O’Connor, PhD, RobertJ. Sokol, MD, Jacquelyn Bertrand, PhD, and \\ José F. Cordero, MD, MPH
}

\begin{abstract}
Alcohol use among women of childbearing age is prevalent in the United States, with approximately 1 in 5 nonpregnant women reporting binge drinking ( 5 or more drinks on any one occasion) and 1 in 25 pregnant women reporting binge drinking. Alcohol use during pregnancy results in a spectrum of adverse outcomes known as fetal alcohol spectrum disorders. Fetal alcohol syndrome (FAS) is one of these disorders. Fetal alcohol syndrome is characterized by specific facial abnormalities and significant impairments in neurodevelopment and physical growth. Early
\end{abstract}

From the National Center on Birth Defects and Developmental Disabilities, Centers for Disease Control and Prevention, Atlanta, Georgia; Department of Psychiatry and Biobehavioral Sciences, University of California at Los Angeles, David Geffen School of Medicine, Los Angeles, California; and Department of Obstetrics and Gynecology, Wayne State University, School of Medicine, Detroit, Michigan.

Information contained in this commentary was derived from a congressionally mandated report, Fetal Alcohol Syndrome: Guidelines for Referral and Diagnosis, which was developed and published in 2004 by the Centers for Disease Control and Prevention in collaboration with the National Task Force on Fetal Alcohol Syndrome and Fetal Alcohol Effect and with representation from the Interagency Coordinating Committee on Fetal Alcohol Syndrome. Authors of this commentary contributed to the development of that report.

Corresponding author: R. Louise Floyd, DSN, RN, Centers for Disease Control and Prevention, $\mathrm{Na}$ tional Center on Birth Defects and Developmental Disabilities, Executive Park Drive, Building 12, Mail-stop E86, Atlanta, GA 30329; e-mail: rlf3@cdc.gov.

(C) 2005 by The American College of Obstetricians and Gynecologists. Published by Lippincott Williams E Wilkins.

ISSN: 0029-7844/05 identification of children with FAS has been shown to enhance their longterm outcomes. In an effort to improve clinical recognition of children with this condition, Centers for Disease Control and Prevention (CDC) was directed by Congress in 2002 to lead the development of uniform diagnostic criteria for FAS and other prenatal alcohol-related conditions. The purpose of this commentary is to provide clinicians a summary of the report released by $\mathrm{CDC}$ describing the current diagnostic criteria for FAS. In addition, advancements have been made in screening and brief interventions for alcohol use disorders in women who have the potential to make significant strides in the prevention of FAS spectrum disorders. Knowledge of the diagnostic criteria for FAS can lead to increased identification of the syndrome in infants and children and the provision of appropriate medical and support services. Screening for and intervening with women at risk for an alcohol-exposed pregnancy can prevent FAS and other fetal alcohol spectrum disorders.

P renatal alcohol exposure is regarded as a leading preventable cause of birth defects and developmental disabilities in the United States, ${ }^{1}$ with fetal alcohol syndrome (FAS) being the most commonly known condition associated with in utero alcohol exposure. In February 2005, the U.S. Surgeon General issued an updated Advisory on Alcohol Use and Pregnancy. The ad- visory recommended that pregnant women should not drink alcohol, that a pregnant woman who had already consumed alcohol during her pregnancy should stop drinking to minimize further risk, and that a woman who is considering pregnancy or who might become pregnant should also abstain from alcohol (http://www.hhs.gov/ surgeongeneral/pressreleases/ sg02222005.html). The Institute of Medicine $(\mathrm{IOM})^{2}$ released a report in 1996 containing broadly defined diagnostic criteria for FAS, partial FAS, alcohol-related birth defects, and alcohol-related neurodevelopment disorder. The report recommended further research to evaluate the utility, reliability, and validity of the proposed method of classifying and diagnosing conditions resulting from prenatal alcohol exposure. In 2002, noting the lack of uniformity in the diagnostic criteria being used to address prenatal alcohol-related conditions in children in the United States, Congress directed the Centers for Disease Control and Prevention (CDC) to establish uniform diagnostic criteria for FAS and other prenatal alcohol-related conditions in collaboration with the National Task Force on Fetal Alcohol Syndrome and Fetal Alcohol Effect, and other federal and nonfederal partners, including the National Institute on Alcohol Abuse and Alcoholism and the Substance Abuse 
and Mental Health Services Administration. A scientific working group comprising medical and behavioral experts in the field of fetal alcohol syndrome was convened to assist and guide the development of the diagnostic guidelines. In July 2004, CDC released a report containing diagnostic criteria for FAS, as well as recommendations for prevention of alcohol-exposed pregnancies. $^{3}$ There is currently a general consensus that prenatal alcohol exposure results in a wide range of adverse effects that, taken as a whole, have been called fetal alcohol spectrum disorders. ${ }^{4}$ The CDC-published report, "Fetal Alcohol Syndrome: Guidelines for Referral and Diagnosis," endorsed by The American College of Obstetricians and Gynecologists (ACOG) and the American Academy of Pediatrics (AAP), acknowledged the lack of scientific evidence available at that time to support specific diagnostic criteria for prenatal alcohol-related conditions beyond FAS and the need to continue to build the scientific evidence needed to assign diagnostic criteria to other prenatal alcoholrelated conditions such as alcoholrelated neurodevelopment disorder. The purpose of this Current Commentary is to summarize briefly the FAS diagnostic criteria contained in the mandated report and the recommended current best practices for identifying and intervening with women at risk for an alcohol-exposed pregnancy.

\section{RECOGNITION OF FETAL ALCOHOL SYNDROME}

The defining characteristics outlined in the expert report released by CDC include those summarized in the box "Brief Outline of Diagnostic Criteria for Fetal Alcohol Syndrome." The full report describing the scientific evidence supporting the selection of the charac- teristics and accompanying measurements that constitute the clinical thresholds for diagnosis can be found at http://www.cdc.gov/ ncbddd/fas/documents/fas_guidelines_accessible.pdf.

Evidence supports the need for early recognition of FAS to reduce the secondary conditions that often accompany maturation. They include school failure, difficulties with peers, conduct problems, and mental health disorders. ${ }^{5}$ Recognition of FAS is made difficult in the newborn period because the pattern of facial dysmorphia characterizing the condition can be subtle,

Therefore, identifying

women who are at high risk for an alcohol-exposed pregnancy and intervening with them before conception is an essential

$$
\begin{gathered}
\text { strategy for preventing } \\
\text { alcohol-exposed } \\
\text { pregnancies. }
\end{gathered}
$$

and the associated central nervous system deficits might not be clearly manifested. However, under-recognition of FAS has been reported in infants born to women known to have abused alcohol during pregnancy when the findings of physician examiners familiar with the condition were compared with the findings of the infants' primary physicians. Reasons cited for under-recognition of FAS in infants include inadequate knowledge of the mother's prenatal drinking patterns and the examining physicians' lack of comfort and experience with the disorder. ${ }^{6}$ Prevalence rates can vary greatly depending on the methods used for case identification, the geographic area, and the racial and ethnic characteristics of the population. For example, one CDC study reported prevalence rates ranging from 0.3 to 1.5 per 1,000 live births in the states of Arizona, Colorado, New York, and Alaska, with highest rates occurring in American Indian and Alaskan Natives and African Americans. ${ }^{7}$ Another report combining studies using a variety of methodologies reported a prevalence rate of $0.5-$ 2.0 cases per 1,000 live births. ${ }^{8}$ Some practitioners rarely encounter a case of FAS. Knowledge of the most salient clinical characteristics of the condition may trigger recognition, particularly in cases where prenatal alcohol exposure is confirmed or suspected.

\section{SCREENING AND INTERVENING WITH WOMEN AT RISK FOR AN ALCOHOL-EXPOSED PREGNANCY}

Centers for Disease Control and Prevention studies report escalating rates of binge drinking in women of childbearing age from 1999 through 2001, with increases in the number of binge drinking episodes per person during that same time period. ${ }^{9}$ Statistics from the 2003 National Survey on Drug Use and Health reported that, among pregnant women aged 15-44 years, 9.8\% used alcohol and $4.1 \%$ reported binge drinking in the month before the survey. Among nonpregnant women of childbearing age, 53\% reported alcohol use and 23\% reported binge drinking. ${ }^{10}$ A number of studies have shown that, as the maternal alcohol intake increases, there is a corresponding increase in the adverse effects observable in the fetus. ${ }^{2}$ Although alcohol dose may not be the sole contributing factor 


\section{BRIEF OUTLINE OF DIAGNOSTIC CRITERIA FOR FETAL ALCOHOL SYNDROME}

\section{Facial Dysmorphia}

Based on racial norms, individual exhibits all 3 characteristic facial features:

I. Smooth philtrum (University of Washington Lip-Philtrum Guide rank 4 or $5^{*}$ )

II. Thin vermillion border (University of Washington Lip-Philtrum Guide rank 4 or 5)

III. Small palpebral fissures (at or below 10th percentile)

\section{Growth Problems}

I. Confirmed prenatal or postnatal height or weight, or both, at or below the 10th percentile, documented at any one point in time (adjusted for age, sex, gestational age, and race or ethnicity).

\section{Central Nervous System Abnormalities}

I. Structural

A. Head circumference (OFC) at or below 10th percentile adjusted for age and sex.

B. Clinically significant brain abnormalities observable through imaging.

II. Neurological

Neurological problems not due to a postnatal insult or fever, or other soft neurological signs outside normal limits.

III. Functional

Performance substantially below that expected for an individual's age, schooling, or circumstances, as evidenced by:

A. Global cognitive or intellectual deficits representing multiple domains of deficit significant developmental delay in younger children) with performance below the 3 rd percentile (2 standard deviations below the mean for standardized testing), or

B. Functional deficits below the 16 th percentile (1 standard deviation below the mean for standardized testing) in at least 3 of the following domains:

1. Cognitive or developmental deficits or discrepancies

2. Executive functioning deficits

3. Motor functioning delays

4. Problems with attention or hyperactivity

5. Social skills

6. Other, such as sensory problems, pragmatic language problems, and memory deficits.

\section{Criteria for Fetal Alcohol Syndrome Diagnosis}

Requires all 3 of the following findings:

I. Documentation of all 3 facial abnormalities (smooth philtrum, thin vermillion border, and small palpebral fissures)

II. Documentation of growth deficit

III. Documentation of central nervous system (CNS) abnormality

* University of Washington Lip-Philtrum Guide http://depts.washington.edu/fasdpn

Source: BertrandJ et al. Fetal alcohol syndrome: guidelines for referral and diagnosis. Atlanta (GA): Centers for Disease Control and Prevention; 2004.

to this phenomenon, it is certainly an important one. Approximately $8 \%$ of women of childbearing age are at risk of pregnancy, and half of them are actively trying to become pregnant. However, half of all pregnancies are unplanned and many women will not know they are pregnant during the early weeks of gestation and will continue consuming alcohol at levels that result in the birth of a child with fetal alcohol spectrum disorders. Therefore, identifying women who are at high risk for an alcoholexposed pregnancy and intervening with them before conception is an essential strategy for preventing alcohol-exposed pregnancies. Recently released evidence-based rec- ommendations from the Agency for Healthcare Research Quality ${ }^{11}$ cited screening and brief interventions as the standard of care for intervening in alcohol abuse problems in adults and extended the recommendation to pregnant women as well.

Screening instruments have been developed and validated for 
T-ACE

Tolerance

"How many drinks does it take to make you feel high?"

Annoyed

"Have people annoyed you by criticizing your drinking?"

\section{Cut Down}

"Have you felt you ought to cut down on your drinking?"

\section{Eye Opener}

"Have you ever had a drink first thing in the morning to steady your nerves or get rid of a hangover?"

Scoring: The first question is scored positive if the respondent answers more than 2 drinks. A positive gets a 2 for this question. The last 3 questions are each scored 1 point each if answered affirmatively. A total score of 2 or more is considered positive for risk drinking.

Modified from Sokol RJ et al. The T-ACE questions: practical prenatal detection of risk-drinking. AmJ Obstet Gynecol 1989;160:863-868. Copyright 1989,

with permission from Elsevier.

\section{TWEAK}

T Tolerance: How many drinks can you "hold"?

(Record number of drinks in box at right.)

(For next questions, check box at right for "yes" answers.)

W Have close friends or relatives Worried or Complained about your drinking in the past year?

E Eye-Opener: Do you sometimes take a drink in the morning when you first get up?

A Amnesia (Blackouts): Has a friend or family member ever told you about things you said or did while you were drinking that you could not remember?

$\mathbf{K}(\mathbf{C})$ Do you sometimes feel the need to Cut Down on your drinking?

To score the test, a 7-point scale is used. The tolerance question scores 2 points if a woman can report she can "hold" more than 5 drinks without passing out, and a positive response to the worry question scores 2 points. Each of the last 3 questions scores 1 point for positive responses. A total of 3 or more points indicates the woman is likely to be a heavy or problem drinker.

Source: Russell M. Nerw assessment tools for risk drinking during pregnancy: T-ACE, TWEAK, and others. Alcohol Health Res World. 1994;18:55-61.

use in pregnant women and nonpregnant women. For pregnant women, the T-ACE (4 items that take less than a minute to administer; see box "T-ACE") and the TWEAK (5 items similar to those in the T-ACE; see box "TWEAK") are brief screening instruments that can improve identification of women with alcohol misuse problems in clinical settings better than can clinician questioning alone. ${ }^{3}$

The TWEAK and T-ACE have been found to also be useful in screening nonpregnant populations of women. The same is true of the
AUDIT, a 10-item screener that has a shorter 3-item version, the AUDIT-C. A fuller discussion of all these instruments and populations they have been tested with can be found in the report, Fetal Alcohol Syndrome: Guidelines for Referral and Diagnosis, and by logging on to http:// www.niaaa.nih.gov/publications/ Assesing $\% 20$ Alcohol/index.htm.

\section{INTERVENING WITH WOMEN AT RISK FOR AN ALCOHOL- EXPOSED PREGNANCY}

Given that there is no established safe level of alcohol use for pregnant women, official guidelines have been issued by ACOG and AAP, ${ }^{12}$ the Office of the Surgeon General, and the Department of Health and Human Services, ${ }^{13}$ advising women who are pregnant or who might become pregnant to abstain from alcohol use. For adult women in general, the Dietary Guidelines for Americans ${ }^{13}$ recommend consuming no more than 1 drink per day. However, as cited earlier, many American women engage in binge drinking and increase their risks for a host of problems, including an alcohol-exposed pregnancy. Until recently, a binge episode was defined as 5 or more 
drinks on any one occasion for both men and women. In 2004, owing to the accumulated evidence of the more severe impact on women than on men, at lower rates of consumption and over shorter periods of time, National Institute on Alcohol Abuse and Alcoholism revised the definition of binge drinking for women to 4 or more drinks per occasion. ${ }^{14}$ Recommendations for clinicians regarding assessment of thresholds published by the National Institute on Alcohol Abuse and Alcoholism recommend that any woman who reports drinking more than 7 drinks per week or more than 3 drinks on any given day be further assessed for risk of developing alcohol-related problems. ${ }^{15}$

Numerous studies have documented the efficacy of brief interventions in reducing harmful alcohol use. ${ }^{3}$ When applying these to women at risk for an alcohol-exposed pregnancy, clinical advice and counseling should include feedback regarding the risk posed, discussion of readiness to change, and assistance provided to women in developing strategies and goals for reducing hazardous use of alcohol. Primary outcomes reported among women of childbearing age receiving brief interventions in primary care settings include a $20-25 \%$ reduction in alcohol drinks per week and episodes of binge drinking. ${ }^{16}$ Preconceptional women may benefit from discussions that include pregnancy postponement and effective contraception to avoid an alcoholexposed pregnancy while she attempts to reach goals of alcohol reduction or abstinence. Follow-up should be conducted for every highrisk woman, and any woman who is unable to achieve her drinking goals should receive stepped-up care, including referral to formal treatment programs or community groups that provide support to women seeking to reduce hazardous alcohol use pat- terns. For women who are already pregnant, recent randomized studies report significant reductions in alcohol use after a brief intervention, ${ }^{17}$ as well as improved infant outcomes (O'Connor MJ. Project CARE: helping pregnant women have healthier babies. Invited presentation to the federal Interagency Coordinating Council on Fetal Alcohol Syndrome (ICCFAS), Rockville, MD, December 12, 2003). Fuller discussions of these approaches can be found in Fetal Alcohol Syndrome: Guidelines for Referral and Diagnosis.

In closing, FAS and all other conditions subsumed under the umbrella of fetal alcohol spectrum disorders are entirely preventable as long as a pregnancy is an alcohol-free pregnancy. Useful screening tools are available, and brief advice and counseling are effective interventions for reducing women's risk for an alcohol-exposed pregnancy. Clinicians are encouraged to incorporate alcohol screening into their routine assessment procedures for women of childbearing age (pregnant and nonpregnant) and to provide advice and counseling appropriate to the screening results. Centers for Disease Control and Prevention and ACOG have recently joined forces to develop and disseminate pocket-sized clinical provider materials that address screening and brief intervention for women of childbearing age who are at risk for an alcohol-exposed pregnancy. Consistently, clinicians say it is important to provide them with health education materials containing messages that are clear and brief and do not require the user to navigate algorithms and text. In addition, final analyses of CDC's Project CHOICES, a brief intervention for preconceptional women at high risk for an alcoholexposed pregnancy, is currently underway with intervention content and study results expected out in 2005. Further information on all aspects discussed in this commentary are available at the following Web sites: http://www.cdc.gov/ncbddd/ fas, http://www.niaaa.nih.gov, and http://www.fascenter.samhsa.gov.

\section{REFERENCES}

1. American Academy of Pediatrics. Committee on Substance Abuse and Committee on Children with Disabilities. Fetal alcohol syndrome and alcoholrelated neurodevelopmental disorders. Pediatrics 2000;106:358-61.

2. Stratton K, Howe C, Battaglia F, editors. Fetal alcohol syndrome: diagnosis, epidemiology, prevention, and treatment. Washington, DC: National Academy Press; 1996.

3. Bertrand J, Floyd RL, Weber MK, O'Connor M, Riley EP, Johnson KA, et al. National Task Force on FAS/FAE. Fetal alcohol syndrome: guidelines for referral and diagnosis. Atlanta (GA): Centers for Disease Control and Prevention; 2004.

4. Sokol RJ, Delaney-Black V, Nordstrom B. Fetal alcohol spectrum disorder. JAMA 2003;290:2996-9.

5. Streissguth AP, Bookstein FL, Barr HM, Sampson PD, O'Malley, K, Young JK. Risk factors for adverse life outcomes in fetal alcohol syndrome and fetal alcohol effects. J Dev Behav Pediatr 2004;25: 228-38.

6. Stoler JM, Holmes LB. Under-recognition of prenatal alcohol effects in infants of known alcohol abusing women. J Pediatr 1999;135:430-6.

7. Centers for Disease Control and Prevention. Fetal alcohol syndrome: Alaska, Arizona, Colorado, and New York, 1995-1997. MMWR Morbid Mortal Wkly Rep 2002;51:433-5.

8. May PA, Gossage JP. Estimating the prevalence of fetal alcohol syndrome: a summary. Alcohol Res Health 2001;25: 159-67.

9. Naimi TS, Brewer RD, Mokdad A, Denny C, Serdula MK, Marks JS. Binge drinking among US adults. JAMA 2003;289:70-5.

10. Substance Abuse and Mental Health Services Administration. Results from the 2003 National Survey on Drug Use and Health: National Findings. Rockville (MD): Office of Applied Studies, NSDUH, Series H-25, DHHS Publication No. SMA 04-3964; 2004. Available at: http://webapp.icpsr.umich.edu/ cocoon/SAMHDA-STUDY/04138.xml. Retrieved August 15, 2004.

11. U.S. Preventive Service Task Force. Screening and behavioral counseling 
interventions in primary care to reduce alcohol misuse: recommendation statement. Ann Intern Med 2004;140:554-6.

12. Guidelines for perinatal care. 5th ed. Elk Grove Village (IL): American Academy of Pediatrics; Washington, DC: American College of Obstetricians and Gynecologists; 2002.

13. Dietary guidelines for Americans. U.S. Department of Health and Human Services. U.S. Department of Agriculture. Available at: http://www.healthierus.gov/ dietaryguidelines. Retrieved August 15, 2005.
14. Department of Health and Human Services. National Institute on Alcohol Abuse and Alcoholism. NIAAA council approves definition of binge drinking. NIAAA Newsletter. No. 3. NIH Publication No. 04-5346. Winter 2004. Available at: http://www.niaaa.nih.gov/ publications/Newsletter/winter2004/ Newsletter_Number3.htm. Retrieved August 15, 2005.

15. National Institute on Alcohol Abuse and Alcoholism. Helping patients with alcohol problems: a health practitioner's guide. NIH Publication No. 03-3769; 2003. Available at: http://www.athealth.
com/Practitioner/ceduc/physguide.html. Retrieved August 15, 2005.

16. Manwell LB, Fleming MF, Mundt MP, Stauffacher EA, Barry KL. Treatment of problem alcohol use in women of childbearing age: Results of a brief intervention trial. Alcohol Clin Exp Res 2000; 24:1517-24.

17. Chang G, McNamara TK, Orav EJ, Koby D, Lavigne A, Ludman G, Vincitorio NA, Wilkins-Haug L. Brief interventions for prenatal alcohol use: a randomized trial. Obstet Gynecol 2005;105:991-8.

\section{OBSTETRICS \&}

GYNECOLOGY

\section{Online Access to Obstetrics \& Gynecology}

ACOG Members: Link to full-text articles on the Green Journal web site (www.greenjournal.org) through the Member Access area on www.acog.org. You do not need to activate your online subscription. However, if you go directly to the Green Journal site, you will need to follow the same activation instructions that apply to nonmembers.

Nonmember Subscribers: Activate your online subscription by following these steps:

- On the home page of www.greenjournal.org, select the "Subscriptions" button from the menu bar on the left side of the page.

- At the top of the next page, click "ACTIVATE Your Member or Individual (Nonmember) Subscription."

- On the Activation page, enter your 7-digit ACOG Member ID number and click the "Submit" button. If you are not a member of ACOG, enter your 12-digit customer number. If your customer number is less than 12 digits, you must precede it with zeros for the system to recognize your account. Your ACOG Member ID or customer number can be found on the mailing label of your print journal cover. 